\title{
Evolution of dust properties inside molecular clouds from coreshine modelling
}

\author{
Charlène Lefèvre*, Laurent Pagani, Mika Juvela, Roberta Paladini ${ }^{\dagger}$ \\ Observatoire de Paris \\ E-mail: charlene.lefevre@obspm.fr
}

\begin{abstract}
Using observations to deduce dust properties, grain size distribution, and physical conditions in molecular clouds is a highly degenerate problem. The coreshine phenomenon, a scattering process at 3.6 and $4.5 \mu \mathrm{m}$ that dominates absorption, has revealed its ability to explore the densest parts of clouds. We want to use this effect to constrain the dust parameters and investigate to what extent grain growth (at constant dust mass) inside molecular clouds is able to explain the coreshine observations ( $\sim 100$ sources detected). We aim to find dust models that can explain a sample of Spitzer coreshine data but we also look at the consistency with near-infrared data we obtained for a few clouds. We built a grid of dust models and investigated the key parameters to reproduce the general trend of surface brightnesses and intensity ratios of both coreshine and near-infrared observations with the help of a 3D Monte-Carlo radiative transfer code. The grid parameters allow to investigate the effect of coagulation upon spherical grains up to $5 \mu \mathrm{m}$ in size derived from the DustEm diffuse interstellar medium grains. Fractal dimension, porosity, ices, and a handful of classical grain distributions were also tested. We used the near- and mostly midinfrared intensity ratios as strong discriminants between dust models. We discuss the modeling parameters as well as the influence of the dust properties and the influence of embedded sources.
\end{abstract}

The Life Cycle of Dust in the Universe: Observations, Theory, and Laboratory Experiments 18-22 November, 2013

Taipei, Taiwan

\footnotetext{
* Speaker.

${ }^{\dagger}$ This research has made use of observations from Spitzer Space Telescope and is financially supported by the DIM ACAV and "Région Ile de france".
} 


\section{Introduction}

The life cycle of dust in the interstellar medium includes many steps before leading to planet formation. Among them, the dust content evolves mainly via grain growth inside molecular clouds: by accretion of heavy gas particles on the dust grains that increases the total dust mass [1], and by the presence of sticky ice mantles (volatile species frozen onto the grains [2]) which favors coagulation [3, 4]. Ice mantle formation beyond $A_{v} \sim 3$ mag [5, 6] implies a change in the grain properties throughout molecular clouds. In addition, interstellar grains evolve with time during the prestellar phase. They continue to grow or possibly reach a stationary state in the cloud envelope [4] while, in the densest region, the dust evolution becomes complex in the presence of an embedded object such as Class 0 or Class I protostars [7]. The thermo-mechanical action of the protostellar object will affect the grains by shattering them in the outflows [8] and by evaporating the grain mantles, releasing water (and other species) in the surrounding gas [9], as seen by Herschel [10, 11]. In this context, to infer the molecular cloud stage from the dust properties is a complex problem which starts by understanding the dust grain content. The widespread coreshine effect [12, 13, 14], a mid-infrared scattering by large grains strong enough to be seen in emission in Spitzer data at 3.6 and $4.5 \mu \mathrm{m}$ but still absorption at 5.8 and $8 \mu \mathrm{m}$, is an opportunity to better constrain both cloud structures and dust properties thanks to 3D-multiwavelengths modeling.

\section{Coreshine emergence: a contrast problem}

The coreshine emergence depends on three main parameters: the illumination radiation field, the cloud background field, and the dust properties. We aimed to constrain the two first in order to investigate what kind of dust would be able to explain our observational sample for a given cloud model.

The standard illumination radiation field (ISRF) given by DIRBE maps was used as an input to the Monte Carlo radiative transfer code (CRT [15]) with the appropriate filter and wavelength scaling corrections [14]. The coreshine signal is the combination of the fraction of the radiation field that is scattered towards the observer $\left(\mathrm{I}_{\mathrm{sca}}\right)$ and the radiation coming from behind the cloud, attenuated on its way through the cloud $\left(\mathrm{I}_{\mathrm{back}}\right)$ [16]. The latest contribution cannot be measured from Spitzer observations since the uncertainty on the zodiacal light estimate is of the same order than the $\mathrm{I}_{\text {back }}$ value itself. Therefore, we follow the method proposed by Levenson et al. [17] to remove the stellar contribution and only retrieve the diffuse emission from DIRBE maps. Taking into account the proportion of diffuse interstellar dust on the line of sight behind the cloud [18], we were able to obtain an accurate estimate for $\mathrm{I}_{\text {back }}$. Thus, the emergent coreshine intensity above the surrounding background at both 3.6 and $4.5 \mu \mathrm{m}$ is governed by $\mathrm{I}_{\lambda}=\mathrm{I}_{\text {sca }}+\mathrm{I}_{\text {back }} \times \exp (-\tau)-\mathrm{I}_{\text {back }}$, where $\tau$ is the integrated extinction map obtained from the radiative transfer code for a given dust model. The cloud background field strength is crucial to understand why we are not able to observe coreshine in the Galactic Plane (Fig. 1) as well as at $5.8 \mu \mathrm{m}^{1}$.

In addition to the Galactic Plane contrast problem, dust properties will act on $\mathrm{I}_{\mathrm{sca}}$ and $\tau$ terms which are wavelength dependent. That is why we propose the coreshine intensity ratio $(\mathrm{CSR})=$ $\mathrm{I}_{4.5 \mu \mathrm{m}} / \mathrm{I}_{3.6 \mu \mathrm{m}}$ as discriminant for dust models.

\footnotetext{
${ }^{1}$ Absorption seen in Spitzer cold data at $8 \mu \mathrm{m}$ is partly due to background field strength and partly to the $9.7 \mu \mathrm{m}$ silicate absorption feature wing that intercepts half of the $8 \mu \mathrm{m}$ filter width.
} 


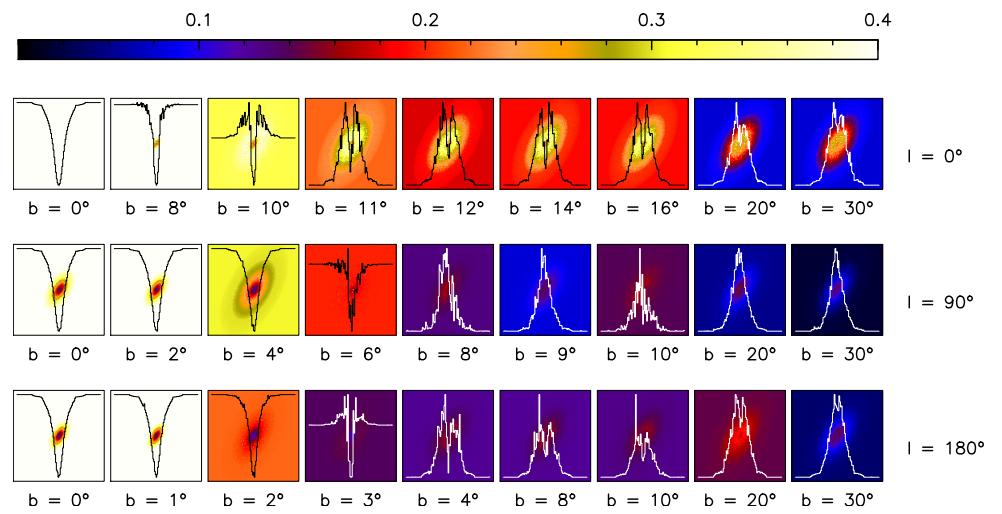

Figure 1: Emergence of coreshine for three galactic longitudes and nine galactic latitudes. For each longitude, the images show the cloud with its background $\left(\mathrm{I}_{3.6 \mu \mathrm{m}}\right.$ $+\mathrm{I}_{\text {back}}$ ). The central profile (cut along $\mathrm{x}$ axis) helps to visualize the coreshine emergence. Fixed color scale images (in $\mathrm{MJy} \mathrm{s}^{-1}$ ) display $\mathrm{I}_{\text {back }}$ variations.

\section{Coreshine ratio}

From observations, we found that the CSR varies between 0.35 and 1.1 in the Galactic regions that present a high occurence of coreshine cases. The highest values are obtained for cores containing an embedded source, which is well-reproduced by adding a mock star in the modeling (Fig. 2 left) that changes locally the illumination field reddening [14].
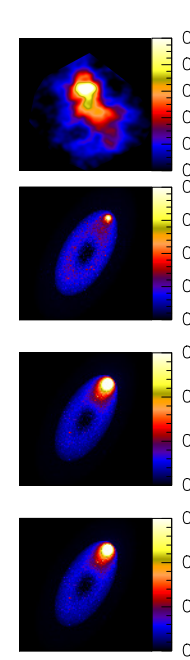
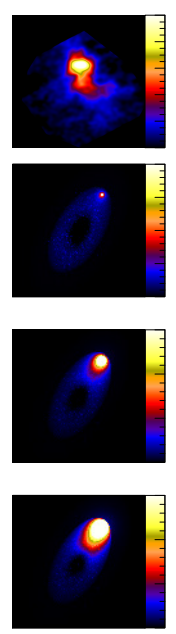
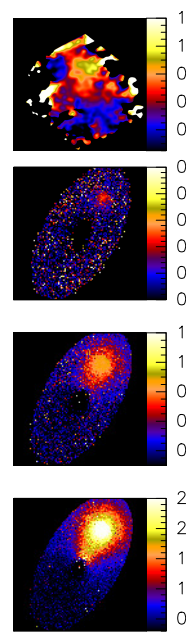

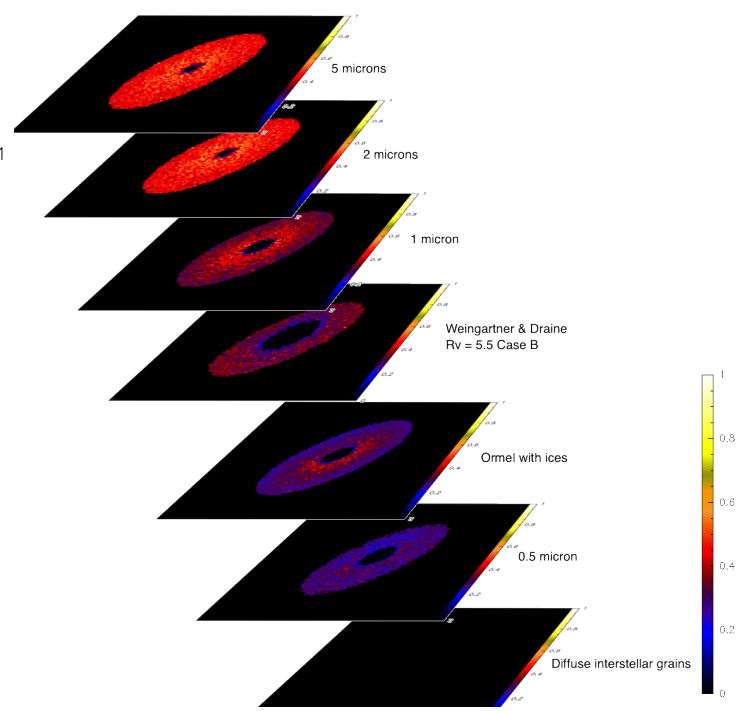

Figure 2: LEFT - With an embedded source - From left to right: IRAC $1(3.6 \mu \mathrm{m})$, IRAC2 (4.5 $\mu \mathrm{m})$, CSR maps. First row: analysis of IRAM04191 Spitzer observations following prescriptions of Lefèvre et al. [14]. Next rows: Modeling obtained with three internal sources ranging from 400 to $2150 \mathrm{~K}$. Color scales are given in MJy sr ${ }^{-1}$. RIGHT - Starless core CSR modeling for different dust properties [4, 19, 20], the given size values correspond to the high exponential cut-off of the whole grain size distribution [14].

For starless cores, where detected, the CSR is always lower than $\sim 0.5$ which is also what is obtained from modeling. Moreover, the CSR starts to saturate around this value with size increase (Fig. 2 right) and grains larger than $2 \mu \mathrm{m}$ are not able to help increase the CSR further. Moreover, $5.8 \mu \mathrm{m}$ wavelength data help to eliminate grain size distributions which contain grains in the 2-5 $\mu \mathrm{m}$ range. 


\section{Conclusion about dust properties}

Analysing the coreshine ratio is a key step to sort the dust properties deep inside the cloud and has been found to be more sensitive to the dust properties than to the cloud structure. The intensity ratio study can then be extended to near-infrared (NIR) wavelengths bringing additional details both on the cloud structure and on the dust properties. For example, while small grains have no impact on the CSR, they will influence the results at NIR wavelengths. By combining several wavelengths, we were able to find suitable dust properties using standard ISRF for starless cores. We also investigated the effect of ices [4], porosity [21] and fractal dimension [22] at mid-infrared wavelengths. Taking into account all the constraints deduced from this grid of models, our next step will be to model in detail the dust content of a real molecular cloud.

\section{References}

[1] Hirashita, H., 2012, MNRAS, 422, 1263

[2] Walmsley, C. M., Flower, D. R.,\& Pineau des Forêts, G., 2004, A\&A, 418, 1035

[3] Ossenkopf, V., 1993, A\&A, 280, 617

[4] Ormel, C. W., Paszon D., Dominik, C.,\& Tielens, A. G. G. M. 2009, A\&A, 502, 845

[5] Whittet, D. C. B., Gerakines, P.A., Hough, J. H., \& Shenoy, S. S. 2001, ApJ, 547, 872

[6] Whittet, D. C. B., Poteet, C. A., Chiar, J. E., et al. 2013, ApJ, 774, 102

[7] André P., Ward-Thompson, D.,\& Barsony, M. 2000, Protostars and Planets IV, 59

[8] Anderl, S., Guillet, V., Pineau des FÃt'rets, G.,\& Flower, D. R. 2013, A\&A, 556, A69

[9] Fraser, H. J., Collings, M. P., McCoustra, M. R. S., \& Williams, D. A. 2001, MNRAS, 327, 1165

[10] Kristensen L. E., van Dishoeck, E. F., Bergin, E. A., et al. 2012, A\&A, 542, A8

[11] van der Tak, F. F. S., Chavarrìa, L., Herpin, F., et al. 2013, A\&A, 554, A83

[12] Pagani, L., Steinacker, J., Bacmann, A., \& Henning, T. 2010, Science, 329, 1622

[13] Paladini, R., Pagani, L., Steinacker, J. et al. 2011, Hunting Coreshine Survey, Spitzer Proposal ID \#80053

[14] Lefèvre, C., Pagani, L., Juvela, M et al., 2014, A\&A, in press

[15] Juvela, M., Padoan, P. 2003, A\&A, 440, 531

[16] Lefèvre, C., Pagani, L., Juvela, M., et al. 2014, in Dust Growth in Star- \& Planet-forming environments, on-line presentations of a conference held in Heidelberg, 22-25 July 2013

[17] Levenson, L. R., Wrght, E. L., \& Johnson, B. D. 2007, ApJ, 666, 34

[18] Lallement, R., Vergely, J.-L., Valette, B., et al. 2014, A\&A, 561, A91

[19] Compiègne, M., Verstraete, L., Jones, A. P., et al. 2011, A\&A, 525, 103

[20] Weingartner, J. C. \& Draine, B.T. 2001, ApJ, 548,296

[21] Ysard, N., Juvela, M., Demyk, K., et al. 2012, A\&A, 542, A21

[22] Min, M., et al. in prep. 